\title{
Clinical impact of HLA class I expression in rectal cancer
}

\author{
Frank M. Speetjens · Elza C. de Bruin • Hans Morreau • Eliane C. M. Zeestraten • \\ Hein Putter · J. Han van Krieken · Maaike M. van Buren • Monique van Velzen • \\ N. Geeske Dekker-Ensink • Cornelis J. H. van de Velde • Peter J. K. Kuppen
}

Received: 5 June 2007 / Accepted: 17 August 2007 / Published online: 15 September 2007

(C) Springer-Verlag 2007

\begin{abstract}
Purpose To determine the clinical impact of human leukocyte antigen (HLA) class I expression in irradiated and non-irradiated rectal carcinomas.

Experimental design Tumor samples in tissue micro array format were collected from 1,135 patients. HLA class I expression was assessed after immunohistochemical staining with two antibodies (HCA2 and $\mathrm{HC} 10$ ).
\end{abstract}

Frank M. Speetjens and Elza C. de Bruin contributed equally to this work.

Cornelis J.H. van de Velde is the Chairperson of the Total Mesorectal Excision Trial.

F. M. Speetjens · E. C. M. Zeestraten · M. M. van Buren · N. G. Dekker-Ensink · C. J. H. van de Velde · P. J. K. Kuppen ( $\square)$ Department of Surgery, Leiden University Medical Center, P.O. Box 9600, 2300 RC Leiden, The Netherlands

e-mail: P.J.K.Kuppen@lumc.nl

F. M. Speetjens

e-mail: f.m.speetjens@lumc.nl

E. C. de Bruin · M. van Velzen

Department of Clinical Oncology,

Leiden University Medical Center, Leiden, The Netherlands

\section{H. Morreau}

Department of Pathology,

Leiden University Medical Center, Leiden, The Netherlands

H. Putter

Medical Statistics and Bioinformatics,

Leiden University Medical Center, Leiden, The Netherlands

J. H. van Krieken

Department of Pathology,

Radboud University Nijmegen Medical Center,

Nijmegen, The Netherlands
Results Tumors were split into two groups: (1) tumors with $>50 \%$ of tumor cells expressing HLA class I (high) and (2) tumors with $\leq 50 \%$ of tumor cells expressing HLA class I (low). No difference in distribution or prognosis of HLA class I expression was found between irradiated and nonirradiated patients. Patients with low expression of HLA class I (15\% of all patients) showed an independent significantly worse prognosis with regard to overall survival and disease-free survival. HLA class I expression had no effect on cancer-specific survival or recurrence-free survival.

Conclusions Down-regulation of HLA class I in rectal cancer is associated with poor prognosis. In contrast to our results, previous reports on HLA class I expression in colorectal cancer described a large population of patients with HLA class I negative tumors, having a good prognosis. This difference might be explained by the fact that a large proportion of HLA negative colon tumors are microsatellite instable (MSI). MSI tumors are associated with a better prognosis than microsatellite stable (MSS). As rectal tumors are mainly MSS, our results suggest that it is both, oncogenic pathway and HLA class I expression, that dictates patient's prognosis in colorectal cancer. Therefore, to prevent confounding in future prognostic analysis on the impact of HLA expression in colorectal tumors, separate analysis of MSI and MSS tumors should be performed.

Keywords Immunology · HLA class I · Prognosis · Rectal cancer $\cdot$ Immunohistochemistry

$\begin{array}{ll}\text { Abbreviations } \\ \text { HLA } & \text { Human leukocyte antigen } \\ \text { MHC } & \text { Major histocompatibility complex } \\ \text { MSI } & \text { Microsatellite instable } \\ \text { MSS } & \text { Microsatellite stable } \\ \text { TMA } & \text { Tissue microarray }\end{array}$


TME Total mesorectal excision

TNM Tumor node metastasis

\section{Introduction}

The immune system is thought to have an important function in controlling tumor growth and eliminating metastasizing tumor cells. The expression of human leukocyte antigen (HLA) class I, presenting tumor-associated antigens on the tumor cell surface, is considered as a prerequisite for an effective $\mathrm{T}$ cell immune response [34]. As a consequence, tumor cells with down-regulated HLA class I expression might escape this immune response, resulting in a selective outgrowth of these tumor cells.

Many studies described HLA class I expression in cancer [10, 17, 22, 33]. Only limited studies have reported on the clinical impact of HLA class I expression in colorectal cancer with contrasting results. Some studies found no significant correlation between staining intensity and survival [1, 19, 20], while others found a prognostic correlation between HLA expression and survival $[18,35]$. The latter two studies had in common that total absence of HLA class I resulted in a favorable prognosis as compared to patients with downregulated expression of HLA class I of tumor cells. The discrepancy between these two studies is, whereas the one described high expression of HLA class I in tumor cells that resulted in a better prognosis as compared to the partial down-regulation of HLA class I [35], the other found the opposite [18]. These studies both analyzed a mixed population of colon and rectal cancer patients. For rectal cancer patients, the clinical impact of HLA class I expression is still unknown. Since HLA class I expression is often absent in microsatellite instable (MSI) tumors [9, 16] and MSI is more frequently observed in right-sided colon tumors than in rectal tumors [30], therefore the results obtained from a mixed population of colon and rectal cancer patients might not hold true for rectal cancer patients.

The purpose of this study was to analyze the clinical relevance of HLA class I expression for rectal cancer patients. In addition to determining the impact of MSI on HLA class I expression, the tumors most at risk for MSI i.e. HLA-negative tumors were examined for MSI by determining the expression of the mismatch repair proteins, mismatch mutL homolog 1 (MLH1) and postmeiotic segregation increased 2 (PMS2), that are most absent in sporadic MSI tumors [7, 36]. Radiotherapy has been described recently to increase cell surface expression of major histocompatibility complex (MHC) class I molecules in a murine colon adenocarcinoma cell line [26]. Therefore, our study also evaluated the effect of radiotherapy on HLA class I expression in rectal cancer patients. For these purposes, HLA class I expression was evaluated in a set of 1,135 formalin-fixed paraffin-embedded rectal cancer specimens. The tumors studied were obtained at the time of surgery from patients of a prospective multicenter trial, who were randomized between standardized preoperative radiotherapy treatment followed by surgery or surgery alone [15].

\section{Materials and methods}

Study population

Patients were obtained from the Dutch TME trial, a multicenter trial that evaluated total mesorectal excision (TME) surgery with or without preoperative radiotherapy $(5 \times 5$ Gray) [15]. Radiotherapeutical, surgical and pathological procedures were standardized and quality-controlled [15, 21]. Tumor staging was determined using the tumor node metastasis (TNM) classification [29]. Patients with the hereditary Lynch syndrome also known as hereditary nonpolyposis colorectal cancer (HNPCC) were excluded from the TME trial. Sufficient formalin-fixed paraffin-embedded tumor material was available for 1,135 Dutch patients. Three $2 \mathrm{~mm}$ cores of each tumor sample were arrayed into tissue microarrays (TMA) as previously described [5].

Immunohistochemistry and microscopic analysis

TMAs [5] were immunohistochemically stained for HLA class I using the mAb antibodies HCA2 and HC10 and the rabbit anti- $\beta 2 \mathrm{~m}$ polyclonal $\mathrm{Ab}$ (A 072; DAKO Cytomation, Glostrup, Denmark). The HCA2 and HC10 antibodies were applied in immunohistochemistry as hybridoma culture supernatant, kindly provided by Prof. J.J. Neefjes from the Netherlands Cancer Institute (Amsterdam, The Netherlands). The reactivity spectrum of HCA2 includes HLA-A (except HLA-A24), HLA-B73 and HLA-C molecules as well as HLA-E, HLA-F and HLA-G antigens [27, 28, 31]. HC10 reacts with HLA-B and HLA-C molecules and HLA-A10, -A28, -A29, -A30, -A31, -A32 and -A33 heavy chains [13, 23, 31, 32]. The immunohistochemical procedures are described in detail elsewhere [18]. All tumor specimens were stained simultaneously to avoid intra-assay variation. Microscopic analysis was assessed by two independent observers (M.M. v. B. and M. v. V.) in a blinded manner. HCA2, $\mathrm{HC} 10$ and $\beta 2 \mathrm{~m}$ stainings were scored in six categories. Essentially, the scoring was divided into quartiles but for tumors with less than $25 \%$ stained cells, there was a distinction made between those with 6-25\% positively stained tumor cells, those with approximately $1-5 \%$ positively stained cells and those with absolute no positively stained tumor cells [3, 11]. Where discrepancies arose between the staining of cores from the same tumor, an 
average of the scores was taken, with confirmation by two observers using a double-headed microscope with a consensus decision taken in all cases. Tissue stromal cells, normal epithelium or lymph follicles served as positive internal controls to ascertain the quality of the staining. Patients were excluded if stromal cells of tumor were not stained for HCA2 or HC10. Twenty-five tumors with negative staining of the stromal cells for HCA2 were excluded. HC10 showed in all tumors staining of the stromal cells. Also TNM stage 0 patients, tumors lost due to technical failure and ineligible patients were excluded, leaving 1,092 tumors in which $\mathrm{HC10}$, and 1,035 in which HCA2 could be evaluated. Combining the results for HCA2 and HC10 staining resulted in 1,008 eligible stage I-IV rectal cancer patients for analyses of clinical impact of HLA class I expression.

Tumors negatively stained for HCA2 and/or HC10 were stained for mismatch repair proteins MLH1 and PMS2. MLH1 and PMS2 are deficient in sporadic MSI tumors. Therefore, the expression of these proteins was used to differentiate MSI and MSS rectal cancers. Tissue stromal cells, normal epithelium or lymph follicles served as positive internal controls when analyzing MLH1, PMS2 expression. The expression of MLH1 and PMS2 was scored positive if tumor cells showed expression, and negative if tumor cells showed no expression of either MLH1 or PMS2, provided that and tissue stromal cells did show expression, indicating microsatellite stable (MSS) and microsatellite instable (MSI) tumors, respectively [7].

\section{Statistical analyses}

All analyses were performed with SPSS statistical software (version 12.0 for Windows, SPSS Inc, Chicago, USA). Mann-Whitney $U, t$ test and $\chi^{2}$-tests were used to compare variables. Kaplan-Meier analyses were performed to analyze patient survival. The entry date for the survival analyses was the time of surgery of the primary tumor. Events for time to local recurrence, distant recurrence, cancer-specific survival, disease-free and overall survival were defined as follows: from time of surgery to time of local disease relapse (for local recurrence), time of distant disease relapse (for distant recurrence), time of disease relapse or death by disease (for cancer specific survival), time of disease relapse or death (for disease free survival) and time of death, respectively (for overall survival). Non-irradiated and irradiated patients were first separately analyzed in univariate analysis and second, variables with a $P$ value of $<0.10$ in the univariate analyses were subjected to a multivariate analysis. Multivariate analysis was performed on the whole group of irradiated and non-irradiated patients with the following variables: HLA class I, randomization for radiotherapy, TNM and circumferential margin. Cox' regression analyses were used to calculate hazard ratios (HR) with $95 \%$ confidence intervals (CI).

\section{Results}

Scoring methods

Several methods are described to analyze HLA class I expression in cancer. The standard is defined by the International HLA and Immunogenetics Workshop (IHIW) [3, 11]. A recent paper describing HLA class I expression in colorectal cancer used an adjusted form of this scoring method [35]. Our scoring was primarily adapted from IWIH, i.e. division into quartiles, but for tumors with less than $25 \%$ stained cells a distinction was made between those with 6$25 \%$ positive tumor cells, those with approximately $1-5 \%$ positive tumor cells and those with absolute no HLA class I positive-stained tumor cells. After scoring and analyzing this method we found that patients in the groups with absolute numbers, $1-5 \%, 6-25 \%$ and $26-50 \%$ HLA class I expression of tumor cells did not differ in prognosis but had a worse prognosis as compared to patients with HLA class I expression in groups with $50-75 \%$ and $>75 \%$ of tumor cells expressing HLA class I. Therefore, we distinguished two categories. These two categories were (1) 0-50\%; and (2) $>50-100 \%$ of tumor cells expressing HLA class I.

HCA-2 and HC10 staining in rectal cancer

Immunohistochemical staining with HCA2 and HC10 antibodies demonstrated strong positive membrane staining of stromal cells and tumor-infiltrating inflammatory cells, indicating the success of the staining. A total of 1,035 and 1,092 tumors were evaluated with HCA2 and HC10; 324 $(65 \%)$ irradiated tumors and $312(58 \%)$ non-irradiated tumors showed at least $50 \%$ of all tumor cells positive for HCA2. Staining with HC10 resulted in 403 (76\%) irradiated tumors and $436(77 \%)$ non-irradiated tumors that showed more than $50 \%$ positive tumor cells. The complete results are shown in Table 1. Representative examples of the immunohistochemical stainings of tumors are displayed in Fig. 1a-f. These results show that about $35 \%$ of irradiated and $42 \%$ of non-irradiated patients showed less than $50 \%$ of the tumor cells expressing HCA2. $\mathrm{HC} 10$ is expressed in less than $50 \%$ of the tumor cells in about $25 \%$ of both irradiated and non-irradiated rectal cancer patients.

\section{Analysis of HLA class I expression in rectal tumors}

Together, the results obtained with HCA2 and $\mathrm{HC} 10$ are expected to reflect HLA class I expression in rectal cancer. In a group of 64 tumors it was studied whether an additional 
Table 1 Most rectal tumors have high numbers of tumor cells positive for $\mathrm{HCA} 2$ or $\mathrm{HC} 10$

\begin{tabular}{lll}
\hline & $\begin{array}{l}\text { Irradiated } \\
\text { patients } N(\%)\end{array}$ & $\begin{array}{l}\text { Non-irradiated } \\
\text { patients } N(\%)\end{array}$ \\
\hline $\begin{array}{ll}\text { HCA2 } \\
\text { High }\end{array}$ & \\
Low & $324(65 \%)$ & $312(58 \%)$ \\
Absence & $142(28 \%)$ & $174(32 \%)$ \\
HC10 & $31(6.2 \%)$ & $52(9.7 \%)$ \\
High & & $436(77 \%)$ \\
Low & $403(76 \%)$ & $116(21 \%)$ \\
Absence & $117(22 \%)$ & $12(2.1 \%)$ \\
\hline
\end{tabular}

Numbers $(N)$ of patients are indicated with percentages shown in parentheses, showing: expression of $\mathrm{HCA} 2$ and $\mathrm{HC} 10$ in more than $50 \%$ of the tumor cells (high), expression in less than $50 \%$ of the tumor cells (low) and total absence (absence)

staining for $\beta 2 \mathrm{~m}$ would better define HLA class I expression. The results of the addition of $\beta 2 \mathrm{~m}$ to $\mathrm{HCA} 2$ and $\mathrm{HC} 10$ were comparable to those obtained with HCA2 and HC10, i.e. only 1 of 64 tumors was differently classified.
Therefore, $\beta 2 \mathrm{~m}$ was not scored in the whole cohort and HLA class I expression was assessed by combining HCA2 and $\mathrm{HC} 10$.

A total of $406(85 \%)$ irradiated and $445(84 \%)$ nonirradiated tumors exhibited expression of at least one of the two markers showing $>50 \%$ positive staining of all tumor cells (further referred to as 'the HLA class I high expression group'). A total of $70(15 \%)$ irradiated and 87 (16\%) non-irradiated tumors showed reduced numbers $(\leq 50 \%)$ of HLA class I positive tumor cells. Only 3 $(0.6 \%)$ irradiated tumors and $8(1.5 \%)$ non-irradiated tumors showed total loss of HLA class I (negative for both HCA2 and HC10). Survival results of patients with total absence of HLA class I on tumor cells did not show significant difference from patients with reduced numbers of HLA class I positive tumor cells. Therefore, these groups were combined and will be further referred to as 'the HLA class I low-expression group'. The complete results are shown in Table 2. The number of patients in the group of the HLA class I high-expression group and the HLA class I low-expression group was equally distributed between irradiated and non-irradiated tumors
Fig. 1 Examples of HCA2 and HC10 immunohistochemical staining of rectal tumors; a-c HCA2, d-f HC10 expression. a, $\mathbf{d}$ Expression of HLA class I in $>50 \%$ tumor cells; $\mathbf{b}, \mathbf{e}$ expression of HLA class I in $<50 \%$ tumor cells; c, f epithelial cells show total absence for HCA2 or $\mathrm{HC} 10$ and only stromal and infiltrative cells show positive staining for HCA2 or $\mathrm{HC} 10$; Original magnification $\times 20$

\section{$\mathrm{HCA} 2$}
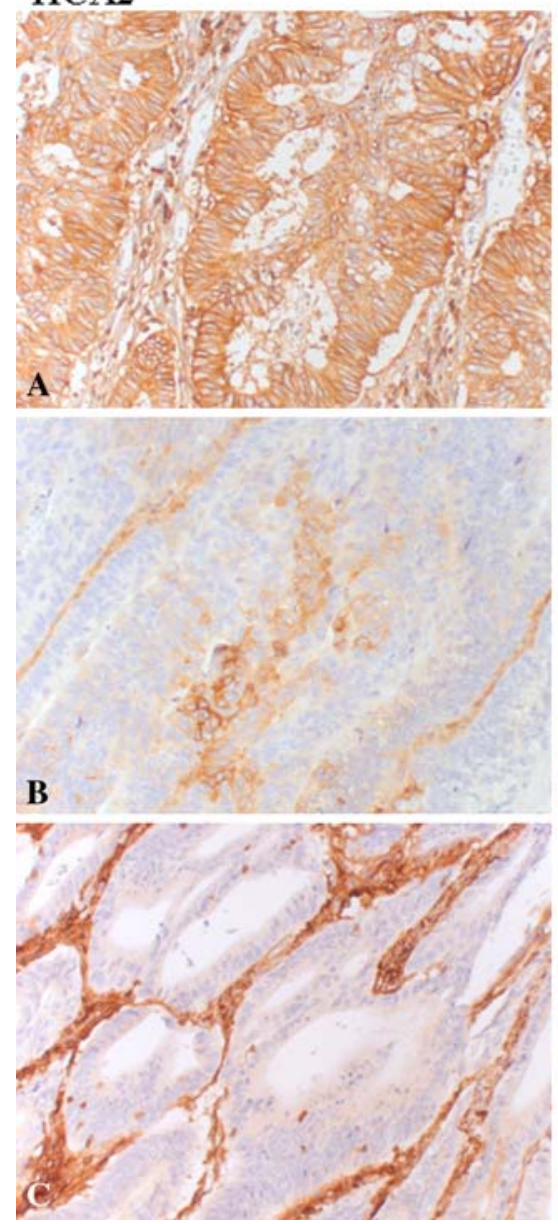

$\mathrm{HC} 10$
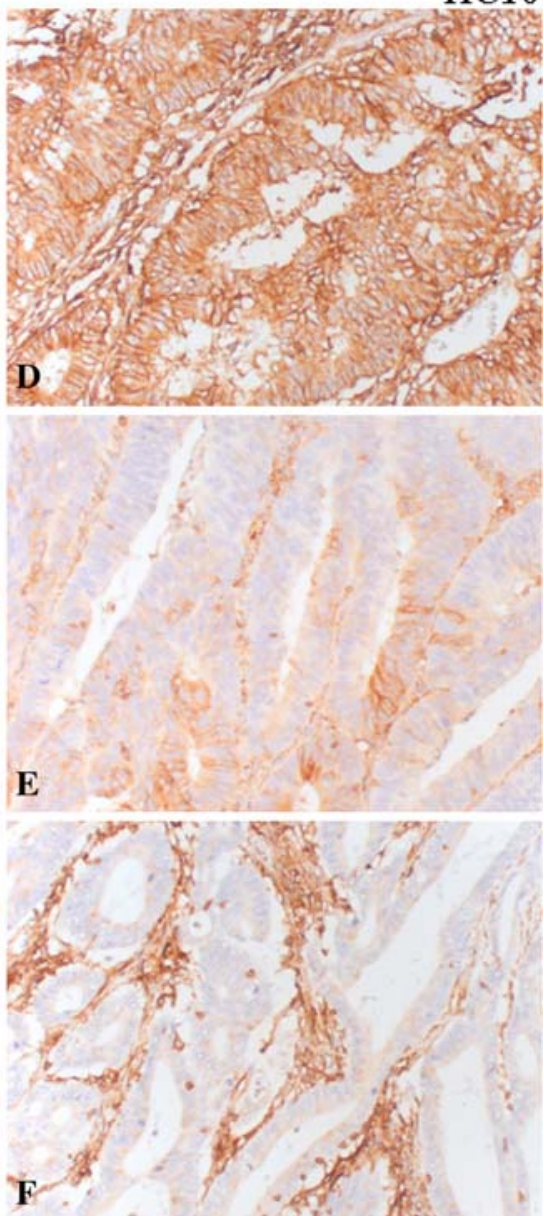
Table 2 Expression of HLA class I in rectal cancer using HCA2 and HC10 antibodies

\begin{tabular}{llllr}
\hline & HCA2 & HC10 & & HLA class I \\
\cline { 3 - 4 } & & High $(\mathrm{N})$ & Low + absence $(N)$ & $N(\%)$ \\
\hline Irradiated & High $(N)$ & 270 & 37 & $406(85 \%)$ \\
& Low + absence $(N)$ & 99 & 70 & $70(15 \%)$ \\
Non-irradiated & High $(N)$ & 277 & 32 & $445(84 \%)$ \\
& Low + absence $(N)$ & 136 & 87 & $87(16 \%)$ \\
\hline
\end{tabular}

Expression of results of $\mathrm{HCA} 2$ and $\mathrm{HC} 10$ staining in a cross table for numbers $(N)$ of irradiated and non-irradiated patients; expression of HCA2 and $\mathrm{HC} 10$ in more than $50 \%$ of the tumor cells (high) versus expression in less than $50 \%$ of the tumor cells (low) is shown. A significant correlation was noted between HCA2 and HC10 staining for both irradiated $\left(\chi^{2}=53.947, P<0.001\right)$ and non-irradiated patients $\left(\chi^{2}=61.257, P<0.001\right)$. The right side of the table displays HLA class I expression estimated on HCA2 and HC10 expression. A total of 406 (85\%) irradiated and 445 (84\%) non-irradiated tumors exhibited expression of at least one of the two markers showing $>50 \%$ positive staining of all tumor cells. A total of 70 ( $15 \%)$ irradiated and $88(16 \%)$ non-irradiated tumors showed reduced numbers $(\leq 50 \%)$ of HLA class I positive tumor cells. The number of patients in the group of the HLA class I high expression group and the HLA class I low expression group was equally distributed between irradiated and nonirradiated tumors $\left(\chi^{2}=0.519, P=0.471\right)$

$\left(\chi^{2}=0.519, P=0.471\right)$, indicating that irradiation had no effect on HLA class I expression in these patients.

HLA class I negative cells and microsatellite instability

It has been described that a majority of MSI colorectal tumors do not express HLA class I, while only a minority of MSS tumors do not express HLA class I $[8,16]$. Therefore, HLA class I negative rectal tumors are the most at risk to be MSI tumors. To evaluate the numbers of sporadic MSI tumors in our study, HCA2 or HC10 negative tumors were analyzed for the expression of PMS2 and MLH1. PMS2 and MLH1 are mismatch repairs proteins that are most frequently absent in MSI sporadic tumors [6]. Of the HLA class I negative tumors only 1 out of 11 tumors did not express PMS2 and MLH1. In the tumor group negative for only 1 of the 2 HLA class I markers, 2 of 81 tumors displayed no PMS2 and MLH1 staining. These results indicate that HLA class I down-regulation is not associated with MSI in rectal cancer and are in accordance with the previous findings that only a very small minority of rectal tumors are MSI $[4,25]$.

HLA class I expression and clinicopathological parameters

The relationship between HLA class I expression and patient/tumor characteristics was assessed (Table 3). The HLA class I expression levels were distributed equally in non-irradiated and irradiated patients with regard to most clinical and pathological parameters. Three significant differences were observed. For the non-irradiated patients, significantly more men appeared in the HLA class I lowexpression group $(P=0.03)$. The group of irradiated tumors with HLA class I low expression contained significantly more stage III and IV tumors $(P=0.01)$ and also more patients with a tumor-positive circumferential resection margin $(P=0.02)$ when compared with tumors with high HLA class I expression.

\section{Expression of HLA class I and clinical prognosis}

Because radiotherapy might influence local tumor recurrences [15], irradiated and non-irradiated tumors were analyzed separately in order to evaluate the impact of HLA class I expression on tumor recurrence and patient survival. The HLA class I expression was not related with distant or local recurrence rates. The patients with low expression of HLA class I had a worse overall survival and diseasefree survival when compared to patients with HLA class I high expression, irrespective of treatment (Fig. 2; overall survival: $P=0.008$ and $P=0.01$; disease free survival: $P=0.01, \quad P=0.006$ in irradiated and non-irradiated patients, respectively). Irradiated patients with low HLA class I expression also had a worse cancer-specific survival $(P=0.003)$. For non-irradiated patients, HLA class I expression had no significant effect on cancer-specific survival (Fig. 2). All results of univariate analysis are shown in Table 4. Univariate analysis showed a better outcome for overall survival and disease-free survival in patients with high HLA class I expression.

Multivariate analysis

Multivariate analysis was performed to identify factors with independent prognostic significance and to calculate hazard ratios (HR). Analyses included TNM, circumferential margin, randomization for preoperative radiotherapy and HLA class I expression (low vs. high HLA class I positive tumor cells) (Table 5). Advanced pathological (TNM) stage and tumor-positive circumferential resection margins retained their strength as independent prognostic factors in these survival analyses. HLA class I expression 
Table 3 Clinicopathological characteristics of irradiated and non-irradiated patients with high or low numbers of HLA class I positive tumor cells

\begin{tabular}{|c|c|c|c|c|c|c|}
\hline & \multicolumn{3}{|c|}{ Non-irradiated patients } & \multicolumn{3}{|c|}{ Irradiated patients } \\
\hline & $\operatorname{High} N=445$ & Low $N=87$ & $P$ value & $\operatorname{High} N=406$ & Low $N=70$ & $P$ value \\
\hline \multicolumn{7}{|l|}{ Gender } \\
\hline Male $(\%)$ & 63 & 75 & 0.03 & 65 & 66 & 0.90 \\
\hline \multicolumn{7}{|l|}{ Age } \\
\hline Median years & 65 & 68 & 0.32 & 65 & 65 & 0.99 \\
\hline \multicolumn{7}{|l|}{ TNM stage $(\%)$} \\
\hline I & 31 & 24 & 0.52 & 33 & 24 & 0.01 \\
\hline II & 27 & 30 & & 30 & 21 & \\
\hline III & 36 & 38 & & 32 & 40 & \\
\hline IV & 5 & 8 & & 5 & 14 & \\
\hline \multicolumn{7}{|l|}{ Circumferential margin } \\
\hline Negative (\%) & 83 & 77 & 0.28 & 86 & 74 & 0.02 \\
\hline \multicolumn{7}{|l|}{ Distant from anal verge $(\%)$} \\
\hline$\geq 10 \mathrm{~cm}$ & 28 & 33 & 0.17 & 27 & 32 & 0.30 \\
\hline $5-10 \mathrm{~cm}$ & 41 & 31 & & 46 & 36 & \\
\hline$<5 \mathrm{~cm}$ & 31 & 36 & & 27 & 32 & \\
\hline \multicolumn{7}{|l|}{ Operation type (\%) } \\
\hline Low anterior resection & 66 & 61 & 0.77 & 65 & 66 & 0.89 \\
\hline Abdomino-perineal resection & 29 & 33 & & 29 & 30 & \\
\hline Hartmann & 5 & 6 & & 6 & 4 & \\
\hline
\end{tabular}

Number $(N)$ of patients with expression of total HLA class I expression in more than 50\% of the tumor cells (high) and expression in less than $50 \%$ of the tumor cells (low)

* Statistical significant $P$ values are in bold

showed independent prognostic value for overall survival and disease-free survival (HR: $1.3, P=0.042$ and HR: $1.4, P=0.006$, respectively), but not for cancer-specific survival.

\section{Discussion}

We showed that rectal cancer patients from the HLA class I low expression group had an independent worse overall and disease-free survival when compared to patients from the HLA class I high-expression group. These data imply that the expression of HLA class I in tumor cells predicts survival for rectal cancer patients. Although significant better cancer-specific survival for irradiated patients with high HLA class I was found in univariate analysis, the predictive value was lost in multivariate analysis. This observation can be explained by the fact that the group with low HLA class I significantly included more stage III/IV and more patients with a positive circumferential margin as compared to the group of patients with high expression of HLA class I. Also no predictive value of HLA class I expression was found with regard to recurrence-free survival of these patients. Therefore, we have no indications that support the notion that better survival of high HLA class I expression is due to the better antigen presenting function of these tumor cells, as has been suggested [18, 35].

In our study, no difference was found between irradiated and non-irradiated patients for HLA class I expression in tumor cells. It has been described that $\gamma$-irradiation induces enhanced peptide production and surface expression of MHC class I in a colorectal mouse tumor cell line [26]. The fact that we could not find more HLA class I expression in irradiated tumors than in non-irradiated tumors indicates that radiotherapy does not induce HLA class I expression in vivo. Immunohistochemistry, however, is less suitable to measure subtle expression changes. Therefore, additional research is required to determine the impact of radiotherapy on expression levels of HLA class $\mathrm{I}$ in human tumors.

In our study, more tumors showed HLA class I downregulation after immunohistochemical staining using HCA2 than using HC10. This difference might be due to differences in reactivity spectrum of both antibodies (see Materials and methods) or to the fact that HLA alleles are differently affected in colorectal cancer. If the latter is the case, our results suggest that HLA A alleles preferentially show downregulation in rectal cancer.

Previous reports evaluated HLA class I expression in mixed patient populations of colon and rectal cancer 
Fig. 2 Examples of KaplanMeier curves showing overall survival and cancer specific survival for irradiated and nonirradiated patients (a-d). Kaplan-Meier curves for overall survival $(\mathbf{a}, \mathbf{b})$ and cancer specific survival (c, d); curves show prognosis for non-irradiated $(\mathbf{a}, \mathbf{c})$ and irradiated patients (b, d) for HLA class I expression in more than $50 \%$ of the tumor cells (high) versus expression in less than $50 \%$ of the tumor cells (low). $P$ value is based on univariate $\log$ rank analyses
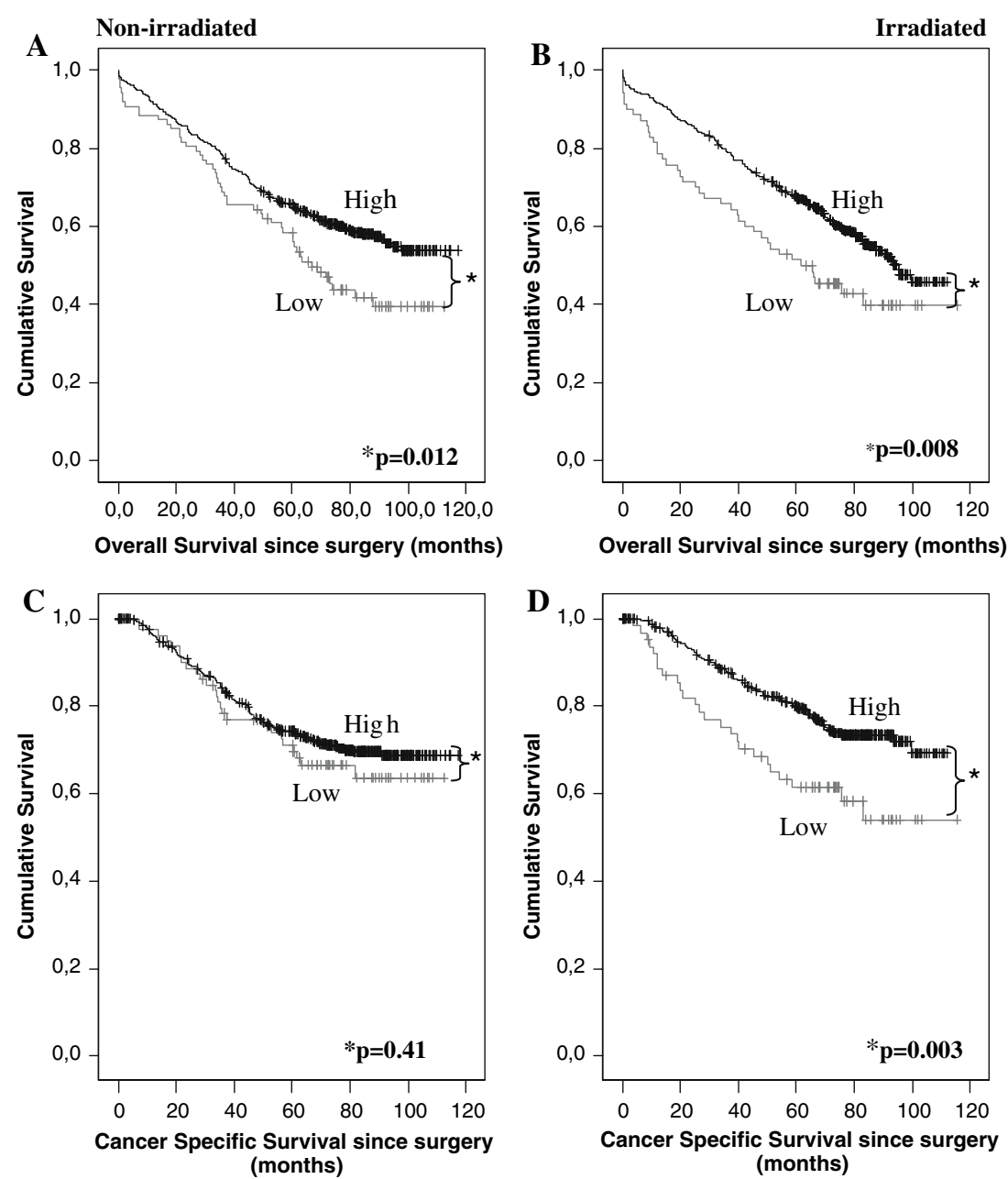

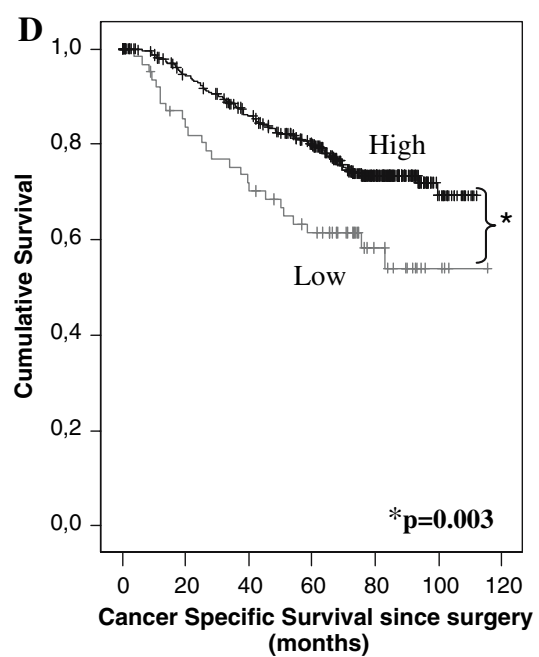

Table 4 Both irradiated and non-irradiated patients with high expression of HLA class I have a better overall, and disease free survival

\begin{tabular}{|c|c|c|c|c|c|c|}
\hline & \multicolumn{3}{|c|}{ Non-irradiated patients } & \multicolumn{3}{|c|}{ Irradiated patients } \\
\hline & High (\%) & Low $(\%)$ & $P$ value & High $(\%)$ & Low $(\%)$ & $P$ value \\
\hline Overall survival & 65.5 & 58.5 & 0.012 & 67.5 & 51.3 & 0.008 \\
\hline Disease free survival & 62.2 & 53.5 & 0.015 & 62.2 & 48.3 & 0.006 \\
\hline Cancer specific survival & 74.3 & 71.4 & 0.41 & 80.1 & 61.8 & 0.003 \\
\hline Local recurrence & 8.9 & 13.7 & 0.22 & 4.7 & 3.2 & 0.72 \\
\hline Distant recurrence & 26.7 & 28.7 & 0.88 & 24.7 & 29.3 & 0.34 \\
\hline
\end{tabular}

Survival and recurrence rates indicated in percentages after 5-years of follow-up for non-irradiated and irradiated patients for HLA class I expression in more than $50 \%$ of the tumor cells (high) versus expression in less than $50 \%$ of the tumor cells (low). $P$ value is based on univariate log rank analyses for overall survival, disease free survival, cancer specific survival, local recurrence and distant recurrence

* Statistical significant $P$ values are in bold

patients [18, 35]. Watson et al. also found in a large group of colorectal cancer patients that patients with low expression of HLA class I had a poor prognosis [35]. However, in contrast to our results, both studies described a substantial population of patients with tumors showing absence of HLA class I. In addition, they described that absence of HLA class I was associated with better prognosis as compared to tumors expressing reduced numbers of HLA class I positive tumor cells. A relatively low number (1.1\%) of HLA class I negative tumors was observed in our cohort of rectal cancer patients only. These patients showed no survival advantage when compared to patients with reduced numbers of HLA class I positive tumor cells. There are several explanations for the discrepancy in the number of HLA class I negative tumors between the study of Watson et al. and ours, like different definition of HLA class I expression, differences in 
Table 5 Multivariate analysis confirms independent better overall, and disease free survival for rectal cancer patients with high expression of HLA class I

\begin{tabular}{|c|c|c|c|c|c|c|}
\hline & \multicolumn{2}{|l|}{ Overall survival } & \multicolumn{2}{|c|}{ Disease free survival } & \multicolumn{2}{|c|}{ Cancer specific survival } \\
\hline & HR $(95 \% \mathrm{CI})$ & $P$ value & $\mathrm{HR}(95 \% \mathrm{CI})$ & $P$ value & $\mathrm{HR}(95 \% \mathrm{CI})$ & $P$ value \\
\hline \multicolumn{7}{|l|}{ HLA } \\
\hline High & 1 & 0.042 & 1 & 0.006 & 1 & 0.653 \\
\hline Low & $1.3(1.0-1.6)$ & & $1.4(1.1-1.8)$ & & $1.1(0.8-1.5)$ & \\
\hline \multicolumn{7}{|c|}{ Randomization } \\
\hline TME & 1 & 0.632 & 1 & 0.214 & 1 & 0.282 \\
\hline $\mathrm{TME}+\mathrm{RT}$ & $1(0.8-1.2)$ & & $0.9(0.7-1.1)$ & & $1.1(0.9-1.5)$ & \\
\hline \multicolumn{7}{|l|}{ TNM } \\
\hline I & 1 & & 1 & & 1 & \\
\hline II & $2.2(1.7-3.0)$ & $<0.001$ & $2.1(1.6-2.8)$ & $<0.001$ & $3.5(2.0-6.1)$ & $<0.001$ \\
\hline III & $3.1(2.4-4.1)$ & $<0.001$ & $3.1(2.3-4.0)$ & $<0.001$ & $9.0(5.4-14.9)$ & $<0.001$ \\
\hline IV & $11.8(8.1-17.1)$ & $<0.001$ & - & - & $50.3(28.5-89.1)$ & $<0.001$ \\
\hline \multicolumn{7}{|l|}{ CRM } \\
\hline Negative & 1 & $<0.001$ & 1 & $<0.001$ & 1 & $<0.001$ \\
\hline Positive & $1.3(1.1-1.5)$ & & $1.8(1.4-2.2)$ & & $1.3(1.1-1.5)$ & \\
\hline
\end{tabular}

Multivariate analysis for cancer specific, overall and disease free survival was performed to identify factors with independent prognostic significance and to calculate hazard ratios $(H R)$ with $95 \%$ confidence intervals $(C I)$ shown in parentheses. HLA class I expression in more than 50\% of the tumor cells (high) versus expression in less than $50 \%$ of the tumor cells (low), total mesorectal excision (TME), Radiotherapy (RT); circumferential margin $(C R M) ; P$ value is based on Cox' regression analyses

* Statistical significant $P$ values and HR are in bold

staining techniques, different patient cohort and number of MSI tumors.

We showed that tumors that do not stain $\mathrm{HC} 10$ can stain positive for HCA2 and thus are still able to present antigens. Therefore, an explanation for the differences with the results of Watson et al. is that we used strict criteria to classify tumors as HLA class I absent (defined as both HCA2 and $\mathrm{HC} 10$ negative) as compared to Watson et al. (defined as negative for $\mathrm{HC} 10$ or negative for $\beta 2 \mathrm{~m}$ instead of negative for both). Another important explanation is that we examined HLA class I expression in a relative more homogeneous population of patients with a rectal tumor, while the other cohorts are more heterogeneous, consisting of both colon and rectal cancer. Although combining results from colon and rectum is generally accepted when predicting prognosis, this might influence results [14].

In colon cancer patients, approximately $50 \%$ of all proximal colon tumors show MSI, whereas almost all distal colon and rectal cancers are MSS tumors [24, 30]. Loss of HLA class I has been described in at least $60 \%$ of all sporadic right-sided MSI colorectal tumors but in only $17 \%$ of MSS right-sided colon tumors loss of HLA class I is found $[8,16]$. In our cohort, only 1 out of 11 HLA negative tumors and 2 out of 81 tumors negative for HCA2 or HC10 did not express MLH1 and PMS2 and were thus likely MSI tumors. This indicates that rectal cancers are mainly MSS tumors, as has previously been described
$[4,14,25]$. Of the multiple mechanisms that have been shown to underlie defects in HLA class I expression in colorectal cancer (mutations in the individual HLA class I genes, mutations in $\beta 2 \mathrm{~m}$ [16], and defects in components of the HLA class I-associated antigen processing machinery (APM) $[2,16])$, only the first will result in allele-specific aberrancies while the other affect total HLA class I expression and may result in total absence in a tumor cell.

These observations imply that a population of colorectal tumors with total absence of HLA class I probably contains a disproportionate large number of MSI tumors when compared to colorectal tumors expressing HLA class I. In addition, MSI colorectal tumors have a better prognosis when compared to MSS colorectal tumors [12, 24]. Therefore, HLA class I negative tumors are more likely to be MSI tumors with a different clinical behavior as compared to MSS colorectal tumors. It is likely that MSI influences prognostic results when considering HLA class I expression in colorectal tumors.

Our results show that HLA class I expression in rectal cancer affects the patient's prognosis. We hypothesize that both oncogenic pathway and HLA class I expression dictate clinical tumor progression. We suggest that in future prognostic studies, analyzing expression of HLA class I or other biomarkers in colorectal cancer, the impact of MSI should be considered. 


\section{References}

1. Benevolo M, Mottolese M, Piperno G et al (2007) HLA-A, -B, -C expression in colon carcinoma mimics that of the normal colonic mucosa and is prognostically relevant. Am J Surg Pathol 31(1):76-84

2. Cabrera CM, Jimenez P, Cabrera T et al (2003) Total loss of MHC class I in colorectal tumors can be explained by two molecular pathways: beta2-microglobulin inactivation in MSI-positive tumors and LMP7/TAP2 downregulation in MSI-negative tumors. Tissue Antigens 61(3):211-219

3. Chew SF, Kanaan C, Tait BD (2007) HLA expression and cancer14th IHIWS immunohistochemistry quality control exercise exchange results. Tissue Antigens 69(Suppl 1):248-251

4. Colombino M, Cossu A, Manca A et al (2002) Prevalence and prognostic role of microsatellite instability in patients with rectal carcinoma. Ann Oncol 13(9):1447-1453

5. de Bruin EC, van de Velde CJ, van de Pas S et al (2006) Prognostic value of apoptosis in rectal cancer patients of the dutch total mesorectal excision trial: radiotherapy is redundant in intrinsically high-apoptotic tumors. Clin Cancer Res 12(21):6432-6436

6. De Jong AE, Morreau H, Van Puijenbroek M et al (2004) The role of mismatch repair gene defects in the development of adenomas in patients with HNPCC. Gastroenterology 126(1):42-48

7. De Jong AE, Van Puijenbroek M, Hendriks Y et al (2004) Microsatellite instability, immunohistochemistry, and additional PMS2 staining in suspected hereditary nonpolyposis colorectal cancer. Clin Cancer Res 10(3):972-980

8. Dierssen JW, de Miranda NF, Ferrone S et al (2007) HNPCC versus sporadic microsatellite-unstable colon cancers follow different routes toward loss of HLA class I expression. BMC Cancer 7(1):33

9. Dierssen JW, de Miranda NF, Mulder A et al (2006) High-resolution analysis of HLA class I alterations in colorectal cancer. BMC Cancer 6:233

10. Ferris RL, Hunt JL, Ferrone S (2005) Human leukocyte antigen (HLA) class I defects in head and neck cancer: molecular mechanisms and clinical significance. Immunol Res 33(2):113-133

11. Garrido F, Cabrer T, Accolla RS et al (1997) HLA and cancer: 12th International Histocompatibility Workshop study. In: Charron D (ed) Genetic diversity of HLA. Functional and medical implication. Proceedings of twelfth international histocompatibility workshop and conference, vol II. EDK, Sevres, France, pp 445-452

12. Gryfe R, Kim H, Hsieh ET et al (2000) Tumor microsatellite instability and clinical outcome in young patients with colorectal cancer. N Engl J Med 342(2):69-77

13. Hutter H, Hammer A, Blaschitz A et al (1996) Expression of HLA class I molecules in human first trimester and term placenta trophoblast. Cell Tissue Res 286(3):439-447

14. Iacopetta B (2002) Are there two sides to colorectal cancer? Int J Cancer 101(5):403-408

15. Kapiteijn E, Marijnen CA, Nagtegaal ID et al (2001) Preoperative radiotherapy combined with total mesorectal excision for resectable rectal cancer. N Engl J Med 345(9):638-646

16. Kloor M, Becker C, Benner A et al (2005) Immunoselective pressure and human leukocyte antigen class I antigen machinery defects in microsatellite unstable colorectal cancers. Cancer Res 65(14):6418-6424

17. Madjd Z, Spendlove I, Pinder SE et al (2005) Total loss of MHC class I is an independent indicator of good prognosis in breast cancer. Int J Cancer 117(2):248-255

18. Menon AG, Morreau H, Tollenaar RA et al (2002) Down-regulation of HLA-A expression correlates with a better prognosis in colorectal cancer patients. Lab Invest 82(12):1725-1733
19. Moller P, Koretz K, Schlag P et al (1991) Frequency of abnormal expression of HLA-A,B,C and HLA-DR molecules, invariant chain, and LFA-3 (CD58) in colorectal carcinoma and its impact on tumor recurrence. Int J Cancer Suppl 6:155-162

20. Moller P, Momburg F, Koretz K et al (1991) Influence of major histocompatibility complex class I and II antigens on survival in colorectal carcinoma. Cancer Res 51(2):729-736

21. Nagtegaal ID, Kranenbarg EK, Hermans J et al (2000) Pathology data in the central databases of multicenter randomized trials need to be based on pathology reports and controlled by trained quality managers. J Clin Oncol 18(8):1771-1779

22. Ogino T, Shigyo H, Ishii H et al (2006) HLA class I antigen downregulation in primary laryngeal squamous cell carcinoma lesions as a poor prognostic marker. Cancer Res 66(18):9281-9289

23. Perosa F, Luccarelli G, Prete M et al (2003) Beta 2-microglobulinfree HLA class I heavy chain epitope mimicry by monoclonal antibody HC-10-specific peptide. J Immunol 171(4):1918-1926

24. Popat S, Hubner R, Houlston RS (2005) Systematic review of microsatellite instability and colorectal cancer prognosis. J Clin Oncol 23(3):609-618

25. Qiu H, Sirivongs P, Rothenberger M et al (2000) Molecular prognostic factors in rectal cancer treated by radiation and surgery. Dis Colon Rectum 43(4):451-459

26. Reits EA, Hodge JW, Herberts CA et al (2006) Radiation modulates the peptide repertoire, enhances MHC class I expression, and induces successful antitumor immunotherapy. J Exp Med 203(5):1259-1271

27. Seitz C, Uchanska-Ziegler B, Zank A et al (1998) The monoclonal antibody HCA2 recognises a broadly shared epitope on selected classical as well as several non-classical HLA class I molecules. Mol Immunol 35(13):819-827

28. Sernee MF, Ploegh HL, Schust DJ (1998) Why certain antibodies cross-react with HLA-A and HLA-G: epitope mapping of two common MHC class I reagents. Mol Immunol 35(3):177-188

29. Sobin LH, Fleming ID (1997) TNM Classification of Malignant Tumors, 5th edn. Union Internationale Contre le Cancer and the American Joint Committee on Cancer. Cancer 80(9):1803-1804

30. Soreide K, Janssen EA, Soiland H et al (2006) Microsatellite instability in colorectal cancer. Br J Surg 93(4):395-406

31. Stam NJ, Spits H, Ploegh HL (1986) Monoclonal antibodies raised against denatured HLA-B locus heavy chains permit biochemical characterization of certain HLA-C locus products. J Immunol 137(7):2299-2306

32. Stam NJ, Vroom TM, Peters PJ et al (1990) HLA-A- and HLAB-specific monoclonal antibodies reactive with free heavy chains in western blots, in formalin-fixed, paraffin-embedded tissue sections and in cryo-immuno-electron microscopy. Int Immunol 2(2):113-125

33. Vitale M, Pelusi G, Taroni B et al (2005) HLA class I antigen down-regulation in primary ovary carcinoma lesions: association with disease stage. Clin Cancer Res 11(1):67-72

34. Wallich R, Bulbuc N, Hammerling GJ et al (1985) Abrogation of metastatic properties of tumour cells by de novo expression of $\mathrm{H}-2 \mathrm{~K}$ antigens following $\mathrm{H}-2$ gene transfection. Nature 315(6017):301-305

35. Watson NF, Ramage JM, Madjd Z et al (2006) Immunosurveillance is active in colorectal cancer as downregulation but not complete loss of MHC class I expression correlates with a poor prognosis. Int J Cancer 118(1):6-10

36. Young J, Simms LA, Biden KG et al (2001) Features of colorectal cancers with high-level microsatellite instability occurring in familial and sporadic settings: parallel pathways of tumorigenesis. Am J Pathol 159(6):2107-2116 